\title{
Mesoscopic non-equilibrium thermodynamics approach to non-Debye dielectric relaxation
}

\author{
Humberto Híjar* and Iván Santamaría-Holek ${ }^{\dagger}$ \\ Facultad de Ciencias, Universidad Nacional Autónoma de México, \\ Circuito Exterior de Ciudad Universitaria, 04510, D. F., México
}

J. G. Méndez-Bermúdez

Instituto de Física, B. Universidad Autónoma de Puebla. Av. San Claudio y 18 Sur, 72570, Puebla, México

\begin{abstract}
Mesoscopic non-equilibrium thermodynamics is used to formulate a model describing nonhomogeneous and non-Debye dielectric relaxation. The model is presented in terms of a FokkerPlanck equation for the probability distribution of non-interacting polar molecules in contact with a heat bath and in the presence of an external time-dependent electric field. Memory effects are introduced in the Fokker-Planck description through integral relations containing memory kernels, which in turn are used to establish a connection with fractional Fokker-Planck descriptions. The model is developed in terms of the evolution equations for the first two moments of the distribution function. These equations are solved by following a perturbative method from which the expressions for the complex susceptibilities are obtained as a functions of the frequency and the wave number. Different memory kernels are considered and used to compare with experiments of dielectric relaxation in glassy systems. For the case of Cole-Cole relaxation, we infer the distribution of relaxation times and its relation with an effective distribution of dipolar moments that can be attributed to different segmental motions of the polymer chains in a melt.
\end{abstract}

PACS numbers:

Keywords: Dielectric relaxation, mesoscopic non-equilibrium thermodynamics, non-Markovian processes

\section{INTRODUCTION}

Relaxation processes in complex systems such as glass-forming liquids, polymeric melts, liquid crystals and even lipid membranes is a very active area of research ${ }^{1-11}$. They have been studied using different techniques ranging from

* Fellow of SNI Mexico. E-mail for correspondence: hijar@daad-alumni.de

$\dagger$ Fellow of SNI Mexico. E-mail for correspondence: isholek.fc@gmail.com 
NMR and Brillouin light scattering to dielectric spectroscopy $\stackrel{1.2}{ }$. The variety of relaxation phenomena occurring in these systems are non-Debye and therefore cannot be characterized by a single relaxation time $\mathrm{e}^{3.4}$. These multiscale time relaxations suggest an accumulative effect of different elemental relaxation processes that can be associated with collective motions in the system² $\underline{\underline{6}}$.

The average effects of these cooperative mechanisms observed in experiments can be accurately described by means of well known empirical relations such as the Williams-Watts function in time space, or through its conjugated frequency domain (Havrilak-Nagami) function. These functions, and many others that can be found in the literature $\mathrm{e}^{\underline{-14}}$, reflect the specific features of the micro- or mesoscopic relaxation dynamics of each system. The microscopic description of these features may involve the formulation of specific molecule-molecule interaction models that lead to particular results. In view of this, it seems important to have a general theoretical model able to cope with different relaxation processes that may describe, for example, the dielectric relaxation in diverse materials including solids, glass-forming liquids and polymer melts $\frac{14}{}$. These last systems may exhibit, for example, anomalous Kerr effect which can be described in terms of models based on fractional Fokker-Planck equations (FFPE) $)^{7} \underline{\underline{.}}$.

In this work we adopt a mesoscopic point of view for describing the relaxation of a system of non-interacting polar molecules inmersed in a time-dependent external electric field, because at this level of description it is still possible to formulate the mentioned general framework able to cope with different relaxation dynamics. In particular, we use the mesoscopic non-equilibrium thermodynamics (MNET) formalism in order to derive a non-Markovian Fokker-Planck like equation (NMFPE) for the evolution of the probability distribution describing the orientation and position of the molecules. The MNET method uses the entropy produced by the system when it is driven out from equilibrium and is particularly useful for describing the stochastic evolution of systems at the mesoscopic scale ${ }^{15-18}$. The advantage of this formalism lies in the fact that the NMFPE derived has a kinetic origin, and therefore there is no explicit assumption on the specific time correlation properties of the underlying Gaussian stochastic process, contrary to what happens with equations derived from Langevin formalisms 19,20 . Within this framework, linear relations between generalized forces and fluxes occurring in phase space are postulated, which allow us to introduce memory effects accounting for the non-instantaneous response of the system to the external perturbations. The effects of the nonMarkovian dynamics on a measurable property of the system like its complex dielectric susceptibility can then be analyzed in a systematic way by considering different memory kernels. In this work we will concentrate mainly on memory kernels decaying as power laws. We will show that these particular functions turn out to be important since they lead to the mentioned FFPE. 
In the section II we will derive the NMFPE for a system of non-interacting polar molecules by using MNET. This equation describes both rotational diffusion and the diffusion of polarization, and we will show that it can be recast into a FFPE when proper memory kernels are considered. In section III we will construct the equations for the first two moments of distribution. Subsequently, in Section IV we will present a perturbation procedure which allow for a solution of the obtained dynamic equations, when the system is subjected to an external harmonic electric field superposed to a large static electric field. Consequently, we will obtain a closed expression for the non-homogeneous dielectric susceptibility of the system which incorporates non-Markovian effects, and the effects of the three first moments of the distribution function. These effects will be analyzed systematically in section $\mathrm{V}$ by considering diverse limiting cases and memory functions. Memory kernels decaying as power laws in time will be shown to generate FFPE and response functions of the Cole-Cole type ${ }^{21}$. A comparison with recent experimental results is performed to validate the models derived. In section VI we will propose a model based on a theory of distribution of relaxation times which can be used to give an insight of the physical processes producing power law kernels and, accordingly, FFPE. Finally, we will present some final remarks an discuss the limitations of our model in section VII.

\section{NON-MARKOVIAN DESCRIPTION OF A SYSTEM OF POLAR MOLECULES}

We shall consider a system of $N$ non-interacting polar molecules with dielectric moment, $\vec{p}$, of magnitude $p$, which are in contact with a thermal bath at temperature $T$. For convenience, in the following we will use the unit vector, $\vec{n}=\vec{p} / p$, which represents the orientation of the molecular dielectric moment, and describe the system in terms of a single-molecule probability density. Let $f(\vec{r}, \vec{n}, t) d \vec{r} d \vec{n}$ denote the probability for finding one molecule at a position ranging from $\vec{r}$ to $\vec{r}+d \vec{r}$, and oriented within the range $\vec{n}$ and $\vec{n}+d \vec{n}$, at time $t$.

The normalization condition,

$$
\iint d \vec{r} d \vec{n} f(\vec{r}, \vec{n}, t)=\mathcal{N}, \text { for all } t
$$

where $\mathcal{N}$ is the numerical density of molecules, imposes the following restriction on the time evolution of the probability density function $f, 22,23$

$$
\frac{\partial f}{\partial t}=-\nabla_{i}\left(f V_{i}\right)-\hat{R}_{i}\left(f \Omega_{i}\right) .
$$


Here, $V_{i}$ and $\Omega_{i}$ are the conjugate velocities to $x_{i}$ and $n_{i}$, respectively; $\nabla_{i}$ is the $i$-th spatial derivative; and $\hat{R}_{i}$ is the $i$-th component of the so called rotational operator

$$
\hat{R}_{i}=\varepsilon_{i j k} n_{j} \frac{\partial}{\partial n_{k}}
$$

In this expression $\varepsilon_{i j k}$ represents the Levi-Civitta antisymmetric symbol and summation over repeated indices will be implicit in the next.

Consider now, that an external time-dependent and uniform electric field, $\vec{E}(t)$, acts on the the system. Then, the potential energy of one molecule in the presence of this field, $U(\vec{n} ; t)$, is an explicit function of the orientation vector and also depends on time, $t$, since the electric field, $\vec{E}(t)$, is time-dependent. In first approximation, the potential energy of the system is given by

$$
U(\vec{n} ; t)=-p \vec{n} \cdot \vec{E}(t)
$$

which holds for molecules in which the anisotropy of the electric polarizability is negligible 24 .

The explicit form of Eq. (2) in terms of $f(\vec{r}, \vec{n}, t)$ can be deduced by analyzing the entropy produced by the system during its time evolution. According to the so called Gibbs Entropy Postulate 15,16 , entropy is defined in terms of the probability distribution $f$ by

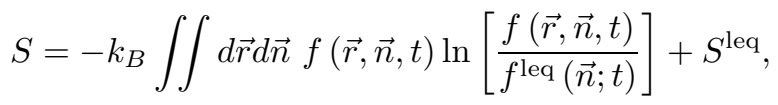

where $k_{B}$ is the Boltzmann constant and $S^{\text {leq }}$ is the local-equilibrium entropy. According to Eq. (3), the corresponding probability density in local-equilibrium is given by canonical distribution function

$$
f^{\text {leq }}(\vec{n} ; t)=\frac{1}{z^{\text {leq }}(T ; t)} \exp \left[-\frac{U(\vec{n} ; t)}{k_{B} T}\right]
$$

where $z^{\text {leq }}$ is the corresponding partition function.

In order to find the entropy production, $\sigma=\partial\left(S-S^{\text {leq }}\right) / \partial t$, we take the time derivative of Eq. (5) from where, 
by using the normalization condition (11) and Eq. (2), we find

$$
\sigma=\frac{1}{T} \iint d \vec{r} d \vec{n}\left[\mu \nabla_{i}\left(f V_{i}\right)+\mu \hat{R}_{i}\left(f \Omega_{i}\right)\right]+k_{B} \iint d \vec{r} d \vec{n} f \frac{\partial}{\partial t} \ln f^{\text {leq }}
$$

where the non-equilibrium chemical potential, $\mu=\mu(\vec{r}, \vec{n}, t)$, has been defined by

$$
\mu(\vec{r}, \vec{n}, t) \equiv k_{B} T \ln \left[\frac{f(\vec{r}, \vec{n}, t)}{f^{\text {leq }}(\vec{n} ; t)}\right]
$$

Now, in accordance with the assumption of local equilibrium, we will consider external fields that evolve in a time-scale larger than the time-scale of fluctuations. Therefore, changes of the local equilibrium distribution are not significant over the short time-scale of the fluctuations, and it can be approximated by $f^{\text {leq }}(\vec{n} ; t) \simeq f^{\text {leq }}(\vec{n} ; 0)$. Consequently, the second term on the right hand side of Eq. (6) vanishes. This approximation is also supported by the fact that later on we will consider electric fields consisting of the superposition of a static, $\vec{E}^{(0)}$, and a time varying, $\vec{E}^{(1)}$, contributions such that the magnitude of the time-dependent superposed field $\overrightarrow{\mathcal{E}}^{(1)}(t)$ is small when compared with the static field $\vec{E}^{(0)}$, that is, we will assume $\vec{E}(t)=\vec{E}^{(0)}+\overrightarrow{\mathcal{E}}^{(1)}(t)$ with $\left|\overrightarrow{\mathcal{E}}^{(1)}\right| /\left|\vec{E}^{(0)}\right|<<1$.

Under this assumption, an integration by parts of Eq. (6) yields the following expression for $\sigma$,

$$
\sigma=-\frac{1}{T} \iint d \vec{r} d \vec{n}\left(f V_{i} \nabla_{i} \mu+f \Omega_{i} \hat{R}_{i} \mu\right)
$$

where we have assumed an infinite system with vanishing probability density at the boundaries in $\vec{r}$-space, and periodic conditions in $\vec{n}$-space.

The entropy production given by Eq. (8) involves the sum of products of the generalized currents, $V_{i}$ and $\Omega_{i}$, and forces, $\nabla_{i} \mu$ and $\hat{R}_{i} \mu$. Thus, following a linear response scheme ${ }^{25}$, we may assume that the currents are proportional to forces and a linear relation for $V_{i}$ and $\Omega_{i}$, in terms of $\nabla_{i} \mu$ and $\hat{R}_{i} \mu$, can be written. For simplicity, we will not consider cross terms in these relations, i.e. we will assume that the generalized force $\nabla_{i} \mu$ is the unique responsible for producing the flux $V_{i}$, and correspondingly for $\hat{R}_{i} \mu$ and $\Omega_{i}$. This assumption is in accordance with the corresponding Onsager-Casimir reciprocal relations given the vectorial character of the vectors involved in the description, see Ref $\underline{\underline{26}}$. In the general case and according to the phenomenology observed, we expect that the response of the system is not instantaneous, and then memory effects should be considered. Following Refs, $\underset{27}{28}$, these effects can be taken into 
account by introducing memory functions in the following linear relationships

$$
\begin{aligned}
& f V_{i}=-\int_{-\infty}^{t} d s K_{i j}(t-s) f(s) \nabla_{j} \mu(s), \\
& f \Omega_{i}=-\int_{-\infty}^{t} d s L_{i j}(t-s) f(s) \hat{R}_{j} \mu(s) .
\end{aligned}
$$

The memory functions $K_{i j}(t)$ and $L_{i j}(t)$, are such that $K_{i j}(t)=L_{i j}(t)=0$, for $t<0$. They describe the delay in the response of the system to forces which vary in time. This delay is always present and is particularly important when forces vary fast in time $\stackrel{25}{\underline{25}}$.

By replacing Eqs. (9) and (10) into Eq. (2), we obtain

$$
\frac{\partial f}{\partial t}=\nabla_{i} \int_{-\infty}^{t} d s \varphi_{i j}(t-s) \nabla_{j} f(s)+\hat{R}_{i} \int_{-\infty}^{t} d s \phi_{i j}(t-s)\left[\hat{R}_{j} f(s)+\frac{f(s)}{k_{B} T} \hat{R}_{j} U\right]
$$

where for notation convenience, we have introduced the modified memory functions $\varphi_{i j}(t)=k_{B} T K_{i j}(t)$, and $\phi_{i j}(t)=$ $k_{B} T L_{i j}(t)$. In order to obtain Eq. (11), we have also used the relations

$$
f \nabla_{i} \mu=k_{B} T \nabla_{i} f, \quad \text { and } \quad f \hat{R}_{i} \mu=k_{B} T \hat{R}_{i} f+f \hat{R}_{i} U
$$

which follow directly from Eq. (7).

Eq. (11) is the desired non-Markovian FP equation for the probability distribution function $f$. It provides a complete probabilistic description of the system at the mesoscopic level and constitutes a generalization of previous equations obtained in the literature for systems with Debye relaxation dynamics 22,29 . The memory kernels $\varphi_{i j}(t)$ and $\phi_{i j}(t)$, characterize the translational and rotational diffusion processes in the system, respectively. For anisotropic molecules such as rigid polymers in solution, the translational diffusion tensor depends in general on the orientational degrees of freedom $n_{i} \underline{24}$. Here, for simplicity, we will restrict ourselves to consider isotropic translational and rotational diffusion processes, i.e. $\varphi_{i j}(t)=\varphi(t) \delta_{i j}$, and $\phi_{i j}(t)=\phi(t) \delta_{i j}$. Consequently, our results will be expected to describe better the dynamics of slightly anisotropic polar molecules. Within this approximation Eq. (11) can be recast in the final 
form

$$
\frac{\partial f}{\partial t}=\nabla^{2} \int_{-\infty}^{t} d s \varphi(t-s) f(s)+\hat{R}_{i} \int_{-\infty}^{t} d s \phi(t-s)\left[\hat{R}_{i} f(s)+\frac{f(s)}{k_{B} T} \hat{R}_{i} U\right]
$$

In addition, notice that we have restricted ourselves to consider memory kernels which only depend on time, and accordingly, we have neglected the possible non-local effects from interactions at different points in space.

In the following sections, Eq. (13) will be used to obtain a dynamical model for the time evolution of the average polarization $P_{i}$ and its associated order parameter, the quadrupole moment $S_{i j}$.

\section{A. Homogeneous Approximation and Fractional Fokker-Planck Equation}

Diverse interesting limiting cases can be obtained from Eq. (13). Firstly, we will consider the so called homogeneous $\operatorname{approximation}^{29}$, in which a simplified description in terms of the reduced orientational probability distribution,

$$
g(\vec{n}, t)=\int d \vec{r} f(\vec{r}, \vec{n}, t)
$$

is given. Averaging Eq. (13) over space coordinates, the corresponding non-Markovian FP equation for $g$ is found to be

$$
\frac{\partial g}{\partial t}=\hat{R}_{i} \int_{-\infty}^{t} d s \phi(t-s)\left[\hat{R}_{i} g(s)+\frac{g(s)}{k_{B} T} \hat{R}_{i} U\right]
$$

Clearly, moments of the reduced distribution $g$ will be uniform time-dependent quantities. Therefore, in the homogeneous approximation spatial diffusion of these moments is ignored and the properties obtained from Eq. (14) must be considered as volume averages 29 .

It is interesting to notice that Eq. (14) leads to a FFPE when the memory function $\phi(t)$ is conveniently chosen to be a power law. Specifically, let

$$
\phi(t)=\left\{\begin{array}{cc}
\frac{D^{2}}{\Gamma(\alpha-1)}(D t)^{\alpha-2} & \text { for } t>0 \\
0 & \text { for } t<0
\end{array}\right.
$$

where $\Gamma$ represents the gamma function, $\alpha$ is a dimensionless parameter within the range $0<\alpha<1$, and $D$ is a 
constant with units of inverse time that can be identified with the rotational diffusion coefficient of the molecules, as we will show below. It follows directly from Eq. (14) that when the memory function is given by Eq. (15), the orientational distribution obeys the dynamic equation

$$
\frac{\partial g}{\partial t}=\frac{D^{\alpha}}{\Gamma(\alpha-1)} \hat{R}_{i} \int_{-\infty}^{t} d s \frac{1}{(t-s)^{2-\alpha}}\left[\hat{R}_{i} g(s)+\frac{g(s)}{k_{B} T} \hat{R}_{i} U\right]
$$

On the right hand side of the last expression we can identify the generalized fractional derivative operator ${ }^{7,30}$

$$
{ }_{-\infty} D_{t}^{-\nu} g(t)=\frac{1}{\Gamma(\nu)} \int_{-\infty}^{t} d s \frac{g(s)}{(t-s)^{1-\nu}}
$$

for $\nu=\alpha-1$. Thus Eq. (16) can be recast in the form

$$
\frac{\partial g}{\partial t}=D_{-\infty}^{\alpha} D_{t}^{1-\alpha} \hat{R}_{i}\left[\hat{R}_{i} g+\frac{g}{k_{B} T} \hat{R}_{i} U\right]
$$

which is the referred FFPE for the orientational distribution.

It can be noticed that Eq. (17) reduces to the usual Fokker-Planck equation for $\alpha=1$ (normal rotational diffusion),

$$
\frac{\partial g}{\partial t}=D \hat{R}_{i}\left[\hat{R}_{i} g+\frac{g}{k_{B} T} \hat{R}_{i} U\right]
$$

where $D$ can be identified as the rotational diffusion coefficient $\stackrel{24}{4}$. For $0<\alpha<1$, Eq. (17) describes the non-Markovian behavior associated with rotational subdiffusive processes.

An interesting point concerning the applicability of the memory function (15) is worth stressing. The presence of $\Gamma(\alpha-1)$ on the right hand side of Eq. (15) yields a negative memory function for $0<\alpha<1$. This implies that the memory function given by Eq. (15) superposes all the effects prior time $t$ with an intrinsic negative sign. This fact is rather masked by the fractional derivative operator appearing in Eq. (17), and as far as we know it has not been noticed in literature where dynamic equations of the type (17) are postulated for the probability distribution $g$. The presence of the function $\Gamma(\alpha-1)$ is, however, necessary in order to obtain the FFPE, Eq. (16), from a power law decay of the memory function.

Finally, we will consider the case in which both rotational and translational diffusion processes are present and can 
be described by power law decaying memory functions. For the translational kernel we introduce the function

$$
\varphi(t)=\left\{\begin{array}{cc}
\frac{\mathcal{D}_{\beta}}{\Gamma(\beta-1)} t^{\beta-2} & \text { for } t>0 \\
0 & \text { for } t<0
\end{array}\right.
$$

where $0<\beta<1$, is a constant parameter, and $\mathcal{D}_{\beta}$ is a generalized translational diffusion coefficient with units of length square times $t^{-\beta 31}$.

By substituting Eqs. (15) and (18) into Eq. (13), it can be easily shown that the FPE describing the evolution of the probability density $f$ takes the form of a fractional equation,

$$
\frac{\partial f}{\partial t}=\mathcal{D}_{\beta-\infty} D_{t}^{1-\beta} \nabla^{2} f+D^{\alpha}{ }_{-\infty} D_{t}^{1-\alpha} \hat{R}_{i}\left[\hat{R}_{i} f+\frac{f}{k_{B} T} \hat{R}_{i} U\right]
$$

which includes anomalous diffusion through the fractional derivative ${ }_{-\infty} D_{t}^{1-\beta}$. In the limit $\alpha, \beta=1$, the Markovian approximation is recovered.

\section{DYNAMIC EQUATIONS FOR THE MULTIPOLAR MOMENTS}

At the macroscopic level, the description of the system must be given in terms of the moments of the distribution function $f$. For this purpose, Eq. (11) can be used to construct a hierarchy of equations for the multipolar moments associated with the charge distribution. Let us consider the dipole, $P_{i}$, quadrupole, $S_{i j}$, and octupole, $Q_{i j k}$ fields, which are defined by the following averages

$$
\begin{gathered}
P_{i}(\vec{r}, t)=\mathcal{N} \int d \vec{n} f(\vec{r}, \vec{n}, t) p_{i}, \\
S_{i j}(\vec{r}, t)=\frac{1}{2} \mathcal{N}^{2} \int d \vec{n} f(\vec{r}, \vec{n}, t)\left(3 p_{i} p_{j}-p^{2} \delta_{i j}\right), \\
Q_{i j k}(\vec{r}, t)=\frac{1}{2} \mathcal{N}^{3} \int d \vec{n} f(\vec{r}, \vec{n}, t)\left[5 p_{i} p_{j} p_{k}-3 p^{2}\{\mathbf{1} \vec{p}\}_{i j k}\right] .
\end{gathered}
$$


In the last definition, $\{\mathbf{1} \vec{p}\}$ represents the symmetric part of the tensor $\mathbf{1} \vec{p}$, which has the explicit form

$$
\{\mathbf{1} \vec{p}\}_{i j k}=\frac{1}{3}\left(p_{i} \delta_{j k}+p_{j} \delta_{i k}+p_{k} \delta_{i j}\right) .
$$

Multiplying Eq. (11) by $\mathcal{N} p_{i}$ and averaging over the orientational degrees of freedom we obtain

$$
\begin{aligned}
\frac{\partial P_{i}}{\partial t}= & \nabla^{2} \int_{-\infty}^{t} d s \varphi(t-s) P_{i}(s)-2 \int_{-\infty}^{t} d s \phi(t-s) P_{i}(s) \\
& +\frac{2 \mathcal{N} p^{2}}{3 k_{B} T} \int_{-\infty}^{t} d s \phi(t-s) E_{i}(s)-\frac{2}{3 \mathcal{N} k_{B} T} \int_{-\infty}^{t} d s \phi(t-s) S_{i j}(s) E_{j}(s)
\end{aligned}
$$

The first term at the right hand side of the last equation accounts for the diffusion of the polarization whereas the other terms are consequences of the orientational dynamics.

Analogously, multiplying Eq. (11) by $\mathcal{N}^{2}\left(3 p_{i} p_{j}-p^{2} \delta_{i j}\right) / 2$ and averaging over $\hat{n}$, we obtain the dynamic equation for the quadrupolar moment,

$$
\begin{aligned}
\frac{\partial S_{i j}}{\partial t}= & \nabla^{2} \int_{-\infty}^{t} d s \varphi(t-s) S_{i j}(s)-6 \int_{-\infty}^{t} d s \phi(t-s) S_{i j}(s) \\
& +\frac{9 \mathcal{N} p^{2}}{10 k_{B} T} \int_{-\infty}^{t} d s \phi(t-s)[[\vec{P}(s) \vec{E}(s)]]_{i j}-\frac{6}{5 \mathcal{N} k_{B} T} \int_{-\infty}^{t} d s \phi(t-s) Q_{i j k}(s) E_{k}(s) .
\end{aligned}
$$

where $[[\vec{P}(t) \vec{E}(t)]]$ is the symmetric traceless part of the dyad $\vec{P} \vec{E}$ evaluated at time $t$, i.e.

$$
[[\vec{P}(t) \vec{E}(t)]]_{i j}=E_{i}(t) P_{j}(t)+E_{j}(t) P_{i}(t)-\frac{2}{3} E_{k}(t) P_{k}(t) \delta_{i j}
$$

It can be noticed from Eq. (22) that the contraction $Q_{i j k}(t) E_{k}(t)$ yields a symmetric traceless tensor of rank two. Consequently, Eq. (24) is in agreement with the symmetry properties of the order parameter tensor $S_{i j}$. For notation's simplicity, in Eqs. (23) and (24) we have not written the dependence of the fields $P_{i}, S_{i j}$ and $Q_{i j k}$ on $\vec{r}$.

Notice that Eqs. (23) and (24) constitute the first two equations of an infinite recurrence hierarchy which includes the time evolution equation for the octupole moment, $Q_{i j k}$, and higher order moments. The problem of approximating this infinite hierarchy by a finite number of equations is a common feature of diverse systems in non-equilibrium statistical mechanics 32,33 . In the following section we will describe in detail our closure approximation for the hierarchy of 
recurrence equations for $P_{i}, S_{i j}, Q_{i j k}$.

In Eqs. (23) and (24), the moments $P_{i}$ and $S_{i j}$ turn out to be coupled only with moments of next superior order because translational diffusion has been assumed to be isotropic. Otherwise the evolution equation for $P_{i}$ will not only involve $S_{i j}$ but $Q_{i j k}$ as well, while the dynamic equation for $S_{i j}$ will include contributions of $Q_{i j k}$ and the fourth order moment. These contributions have been shown to be important in recent literature for describing the dynamics of anisotropic molecules like those composing liquid crystals materials ${ }^{22}$.

Eqs. (23) and (24) can be reduced to the homogeneous case in which both $P_{i}$ and $S_{i j}$ can be approximated by their position-independent volume averages

$$
P_{i}^{\mathrm{h}}(t)=\mathcal{N} \int d \vec{n} g(\vec{n}, t) p_{i}
$$

and

$$
S_{i j}^{\mathrm{h}}(t)=\frac{1}{2} \mathcal{N}^{2} \int d \vec{n} g(\vec{n}, t)\left(3 p_{i} p_{j}-p^{2} \delta_{i j}\right)
$$

Then, it can be proved that $P_{i}^{\mathrm{h}}$ and $S_{i j}^{\mathrm{h}}$ obey the dynamical equations

$$
\begin{aligned}
\frac{\partial P_{i}^{\mathrm{h}}}{\partial t}= & -2 \int_{-\infty}^{t} d s \phi(t-s) P_{i}^{\mathrm{h}}(s)+\frac{2 \mathcal{N} p^{2}}{3 k_{B} T} \int_{-\infty}^{t} d s \phi(t-s) E_{i}(s) \\
& -\frac{2}{3 \mathcal{N} k_{B} T} \int_{-\infty}^{t} d s \phi(t-s) S_{i j}^{\mathrm{h}}(s) E_{j}(s)
\end{aligned}
$$

and

$$
\begin{aligned}
\frac{\partial S_{i j}^{\mathrm{h}}}{\partial t}= & -6 \int_{-\infty}^{t} d s \phi(t-s) S_{i j}^{\mathrm{h}}(s)+\frac{9 \mathcal{N} p^{2}}{10 k_{B} T} \int_{-\infty}^{t} d s \phi(t-s)\left[\left[\vec{P}^{\mathrm{h}}(s) \vec{E}(s)\right]\right]_{i j} \\
& -\frac{6}{5 \mathcal{N} k_{B} T} \int_{-\infty}^{t} d s \phi(t-s) Q_{i j k}(s) E_{k}(s)
\end{aligned}
$$

respectively. Eqs. (27) and (28) can also be obtained from Eq. (14) for the reduced orientational probability distribution after multiplying and averaging over $\hat{n}$, as it was done in the case of Eqs. (23) and (24). 


\section{PERTURBATION THEORY AND DIELECTRIC RELAXATION}

In the following, we will focus on calculating the response function to a small oscillating field $\vec{E}^{(1)}(t)=\overrightarrow{\mathcal{E}}^{(1)} e^{i \omega t}$, which is superposed to a large static field $\vec{E}^{(0)}$, such that $\mathcal{E}^{(1)} / E^{(0)} \ll 1$. Accordingly, the total field acting on the system is $\vec{E}(t)=\vec{E}^{(0)}+\overrightarrow{\mathcal{E}}^{(1)} e^{i \omega t}$. In this section we shall show that a perturbation theory in terms of the small parameter, $\mathcal{E}^{(1)} / E^{(0)}$, can be used in order to find the frequency and field-dependent dielectric susceptibility of the system.

With this purpose, let $P_{i}^{(0)}, S_{i j}^{(0)}$, etc., denote the electric multipolar moments associated with the static field. It is clear that these quantities relax towards equilibrium by following equations which are completely analogous to Eqs. (23) and (24), with $P_{i}^{(0)}, S_{i j}^{(0)}, Q_{i j k}^{(0)}$ and $E_{i}^{(0)}$, replacing the fields $P_{i}, S_{i j}, Q_{i j k}$ and $E_{i}$, respectively. When both $\vec{E}^{(0)}$ and $\vec{E}^{(1)}(t)$ act on the system, the response to the oscillating field can be identified from the dynamics of the perturbed fields $P_{i}^{(1)}=P_{i}-P_{i}^{(0)}, S_{i j}^{(1)}=S_{i j}-S_{i j}^{(0)}$, etc. ${ }^{25}$. By substracting the equations corresponding to the unperturbed fields from Eqs. (23) and (24), the following expressions are found for the time evolution of the perturbed fields

$$
\begin{aligned}
& \frac{\partial P_{i}^{(1)}}{\partial t}=\nabla^{2} \int_{-\infty}^{t} d s \varphi(t-s) P_{i}^{(1)}(s)-2 \int_{-\infty}^{t} d s \phi(t-s) P_{i}^{(1)}(s) \\
& +\frac{2 \mathcal{N} p^{2}}{3 k_{B} T} \int_{-\infty}^{t} d s \phi(t-s) E_{i}^{(1)}(s) \\
& -\frac{2}{3 \mathcal{N} k_{B} T} \int_{-\infty}^{t} d s \phi(t-s)\left[S_{i j}(s) E_{j}(s)-S_{i j}^{(0)}(s) E_{j}^{(0)}\right] \\
& \frac{\partial S_{i j}^{(1)}}{\partial t}=\nabla^{2} \int_{-\infty}^{t} d s \varphi(t-s) S_{i j}^{(1)}(s)-6 \int_{-\infty}^{t} d s \phi(t-s) S_{i j}^{(1)}(s) \\
& +\frac{9 \mathcal{N} p^{2}}{10 k_{B} T} \int_{-\infty}^{t} d s \phi(t-s)\left[\left[\vec{P}(s) \vec{E}(s)-\vec{P}^{(0)}(s) \vec{E}^{(0)}\right]\right]_{i j} \\
& -\frac{6}{5 \mathcal{N} k_{B} T} \int_{-\infty}^{t} d s \phi(t-s)\left[Q_{i j k}(s) E_{k}(s)-Q_{i j k}^{(0)}(s) E_{k}^{(0)}\right] .
\end{aligned}
$$

For small perturbative fields, $E^{(1)} \ll E^{(0)}$, deviations with respect to $P_{i}^{(0)}, S_{i j}^{(0)}, Q_{i j k}^{(0)}$, etc., are expected to be also 
small. In this case, we can approximate

$$
\begin{gathered}
S_{i j}(t) E_{j}(t)-S_{i j}^{(0)}(t) E_{j}^{(0)} \simeq S_{i j}^{(0)}(t) E_{j}^{(1)}(t)+S_{i j}^{(1)}(t) E_{j}^{(0)}, \\
{\left[\left[\vec{P}(t) \vec{E}(t)-\vec{P}^{(0)}(t) \vec{E}^{(0)}\right]\right]_{i j} \simeq\left[\left[\vec{P}^{(0)}(t) \vec{E}^{(1)}(t)\right]\right]_{i j}+\left[\left[\vec{P}^{(1)}(t) \vec{E}^{(0)}\right]\right]_{i j},} \\
Q_{i j k}(t) E_{k}(t)-Q_{i j k}^{(0)}(t) E_{k}^{(0)} \simeq Q_{i j k}^{(0)}(t) E_{k}^{(1)}(t)+Q_{i j k}^{(1)}(t) E_{k}^{(0)} .
\end{gathered}
$$

Moreover, for a large static field we can assume that the non-perturbed multipoles remain close to their equilibrium time-independent values, i.e. $P_{i}^{(0)}(t) \simeq P_{i}^{\mathrm{eq}}\left(\vec{E}^{(0)}\right) \equiv P_{i}^{\mathrm{eq}}, S_{i j}^{(0)}(t) \simeq S_{i j}^{\mathrm{eq}}\left(\vec{E}^{(0)}\right) \equiv S_{i j}^{\mathrm{eq}}, Q_{i j k}^{(0)}(t) \simeq Q_{i j k}^{\mathrm{eq}}\left(\vec{E}^{(0)}\right) \equiv$ $Q_{i j k}^{\mathrm{eq}}$, where, in order to simplify the succeeding discussion, we have omitted to write the dependence of the equilibrium moments on $\vec{E}^{(0)}$. In the following it should be regarded that these quantities are of order zero in the perturbative field.

Finally, in order to close the hierarchy of recurrence equations, we will assume that moments of order $Q_{i j k}$ and higher are not perturbed at all by the time dependent field, and consequently $Q_{i j k}^{(1)}(t) \simeq 0$, for all $t$.

All these approximations yield the following closed system of equations for the time evolution of the perturbed dipole, $P_{i}^{(1)}$, and quadrupole, $S_{i j}^{(1)}$, moments

$$
\begin{aligned}
\frac{\partial P_{i}^{(1)}}{\partial t}= & \nabla^{2} \int_{-\infty}^{t} d s \varphi(t-s) P_{i}^{(1)}(s)-2 \int_{-\infty}^{t} d s \phi(t-s) P_{i}^{(1)}(s) \\
& +\frac{2 \mathcal{N} p^{2}}{3 k_{B} T} \int_{-\infty}^{t} d s \phi(t-s) E_{i}^{(1)}(s) \\
& -\frac{2}{3 \mathcal{N} k_{B} T} \int_{-\infty}^{t} d s \phi(t-s)\left[S_{i j}^{\mathrm{eq}} E_{j}^{(1)}(s)+S_{i j}^{(1)}(s) E_{j}^{(0)}\right],
\end{aligned}
$$




$$
\begin{aligned}
\frac{\partial S_{i j}^{(1)}}{\partial t}= & \nabla^{2} \int_{-\infty}^{t} d s \varphi(t-s) S_{i j}^{(1)}(s)-6 \int_{-\infty}^{t} d s \phi(t-s) S_{i j}^{(1)}(s) \\
& +\frac{9 \mathcal{N} p^{2}}{10 k_{B} T} \int_{-\infty}^{t} d s \phi(t-s)\left\{\left[\left[\vec{P}^{\mathrm{eq}} \vec{E}^{(1)}(s)\right]\right]_{i j}+\left[\left[\vec{P}^{(1)}(s) \vec{E}^{(0)}\right]\right]_{i j}\right\} \\
& -\frac{6}{5 \mathcal{N} k_{B} T} \int_{-\infty}^{t} d s \phi(t-s) Q_{i j k}^{\mathrm{eq}} E_{k}^{(1)}(s) .
\end{aligned}
$$

It can be observed that the solution of Eqs. (31) and (32), gives indeed the perturbed polarization as a linear function of the perturbative field. From this solution the dielectric susceptibility of the system to the small oscillating field can be identified. For this purpose, we consider the Fourier transform of Eqs. (31) and (32), which can be written in the form

$$
\begin{aligned}
& G_{1}(\vec{k}, \omega) P_{i}^{(1)}(\vec{k}, \omega)=2 \phi(\omega)\left\{\chi_{0} \delta_{i j}-\frac{1}{3 \mathcal{N} k_{B} T}\left[S_{i j}^{\mathrm{eq}} E_{j}^{(1)}(\vec{k}, \omega)+S_{i j}^{(1)}(\vec{k}, \omega) E_{j}^{(0)}\right]\right\}, \\
& G_{2}(\vec{k}, \omega) S_{i j}^{(1)}(\vec{k}, \omega)= \frac{27}{10} \chi_{0} \phi(\omega)\left\{\left[\left[\vec{P}^{\mathrm{eq}} \vec{E}^{(1)}(\vec{k}, \omega)\right]\right]_{i j}+\left[\left[\vec{P}^{(1)}(\vec{k}, \omega) \vec{E}^{(0)}\right]\right]_{i j}\right\} \\
&-\frac{6}{5 \mathcal{N} k_{B} T} \phi(\omega) .
\end{aligned}
$$

Here we have introduced the notation $\chi_{0}=\mathcal{N} p^{2} / 3 k_{B} T$, for the static dielectric susceptibility, and $G_{1}(\vec{k}, \omega)$ and $G_{2}(\vec{k}, \omega)$ for the propagators in Fourier space defined by

$$
\begin{aligned}
& G_{1}(\vec{k}, \omega)=i \omega+2 \phi(\omega)+k^{2} \varphi(\omega), \\
& G_{2}(\vec{k}, \omega)=i \omega+6 \phi(\omega)+k^{2} \varphi(\omega) .
\end{aligned}
$$

In Eqs. (33) and (34) we have also introduced the notation $\vec{E}^{(1)}(\vec{k}, \omega)=(2 \pi)^{3} \vec{E}^{(1)}(\omega) \delta(\vec{k})$, for the space-time Fourier transform of the perturbation field.

The frequency-dependent dielectric susceptibility can be identified by solving Eq. (34) for $S_{i j}^{(1)}$, inserting the result 
into Eq. (33), and solving for $P_{i}^{(1)}$. After some algebraic manipulations this procedure finally yields

$$
P_{i}^{(1)}(\vec{k}, \omega)=\chi_{i j}(\vec{k}, \omega) E_{j}^{(1)}(\vec{k}, \omega)
$$

where the susceptibility, $\chi_{i j}(\vec{k}, \omega)$, can be written as the product $\chi_{i j}=M_{i k}^{-1} N_{k j}$, with the following definition for the matrices $M_{i j}$ and $N_{i j}$,

$$
\begin{aligned}
M_{i j}(\vec{k}, \omega)= & G_{1}(\vec{k}, \omega) \delta_{i j}+\frac{p^{2}}{5 k_{B}^{2} T^{2}} G_{2}^{-1}(\vec{k}, \omega) \phi^{2}(\omega)\left(3 E^{(0) 2} \delta_{i j}+E_{i}^{(0)} E_{j}^{(0)}\right), \\
N_{i j}(\vec{k}, \omega)= & \phi(\omega)\left\{2 \chi_{0} \delta_{i j}-\frac{2}{3 \mathcal{N} k_{B} T} S_{i j}^{\mathrm{eq}}+\frac{4}{5 \mathcal{N}^{2} k_{B}^{2} T^{2}} G_{2}^{-1}(\vec{k}, \omega) \phi(\omega) Q_{i j k}^{\mathrm{eq}} E_{k}^{(0)}\right. \\
& \left.+\frac{3 p^{2}}{5 k_{B}^{2} T^{2}} G_{2}^{-1}(\vec{k}, \omega) \phi(\omega)\left[P_{i}^{\mathrm{eq}} E_{j}^{(0)}+P_{k}^{\mathrm{eq}} E_{k}^{(0)} \delta_{i j}-\frac{2}{3} P_{j}^{\mathrm{eq}} E_{i}^{(0)}\right]\right\} .
\end{aligned}
$$

In the following section we will explicitely calculate the complex dielectric susceptibility, $\chi(\vec{k}, \omega)$, for a specific geometry of the external fields and explore the effects of the memory functions, $\phi(\omega)$ and $\varphi(\omega)$, on this quantity.

\section{RESULTS}

In oder to illustrate the effects of the different mechanisms considered in previous sections on the response of the system to the applied fields, in this section we consider a specific geometry in which the static electric field, $\vec{E}^{(0)}$, is directed along the $\hat{e}_{3}$-axis of a Cartesian coordinate system. Furthermore, we will calculate the equilibrium tensors $P_{i}^{\mathrm{eq}}, S_{i j}^{\mathrm{eq}}$ and $Q_{i j k}^{\mathrm{eq}}$, by using the stationary solution of the reduced orientational equation, Eq. (14),

$$
g^{\mathrm{eq}}(\vec{n})=\frac{p E^{(0)}}{4 \pi k_{B} T} \frac{\exp \left(p \vec{n} \cdot \vec{E}^{(0)} / k_{B} T\right)}{\sinh \left(p E^{(0)} / k_{B} T\right)} .
$$

For this probability density and the geometry described above, we obtain the following expressions for the equilib- 
rium averages of the moments entering in Eqs. (38) and (39):

$$
\begin{gathered}
\left(P_{i}^{\mathrm{eq}}\right)=p \mathcal{N}\left(\begin{array}{c}
0 \\
0 \\
L(\xi)
\end{array}\right), \\
\left(S_{i j}^{\mathrm{eq}}\right)=p^{2} \mathcal{N}^{2}\left(1-\frac{3 L(\xi)}{\xi}\right)\left(\begin{array}{rrr}
-1 / 2 & 0 & 0 \\
0 & -1 / 2 & 0 \\
0 & 1 & 0 \\
0 & 1
\end{array}\right), \\
\left(Q_{i j k}^{\mathrm{eq}} E_{k}^{(0)}\right)=p^{3} \mathcal{N}^{3} E^{(0)}\left[\left(1+\frac{15}{\xi^{2}}\right) L(\xi)-\frac{5}{\xi}\right]\left(\begin{array}{rrr}
-1 / 2 & 0 & 0 \\
0 & -1 / 2 & 0 \\
0 & 1
\end{array}\right) .
\end{gathered}
$$

In Eqs. (41)-(43), the dimensionless parameter $\xi \equiv p E^{(0)} / k_{B} T$ quantifies the electric energy of a molecule in the static field relative to the thermal energy, and $L(\xi)=\operatorname{coth}(\xi)-1 / \xi$ represents the Langevin function. As expected, from Eqs. (38), (39) and (41)-(43), we obtain that the matrices $M_{i j}, N_{i j}$ and $\chi_{i j}$ reflect the uniaxial symmetry of the problem and are given by

$$
\left(M_{i j}\right)=\left(\begin{array}{ccc}
M_{\perp} & 0 & 0 \\
0 & M_{\perp} & 0 \\
0 & 0 & M_{\|}
\end{array}\right),\left(N_{i j}\right)=\left(\begin{array}{ccc}
N_{\perp} & 0 & 0 \\
0 & N_{\perp} & 0 \\
0 & 0 & N_{\|}
\end{array}\right) \text {, and }\left(\chi_{i j}\right)=\left(\begin{array}{ccc}
\chi_{\perp} & 0 & 0 \\
0 & \chi_{\perp} & 0 \\
0 & 0 & \chi_{\|}
\end{array}\right) \text {. }
$$

After calculating the expressions for the components $M_{\perp}, M_{\|}, N_{\perp}$ and $N_{\|}$(see the Appendix), the corresponding formulas for the components of the dielectric susceptibility perpendicular and parallel to the static field are found to be

$$
\chi_{\perp}(\vec{k}, \omega)=\frac{\frac{3}{2} \chi_{0}}{\tilde{G}_{1}(\vec{k}, \omega)+\frac{3}{20} \xi^{2} \tilde{G}_{2}^{-1}(\vec{k}, \omega)}\left\{1-\frac{L(\xi)}{\xi}+\tilde{G}_{2}^{-1}(\vec{k}, \omega)\left[1-\left(\frac{\xi}{2}+\frac{3}{\xi}\right) L(\xi)\right]\right\}
$$




$$
\chi_{\|}(\vec{k}, \omega)=\frac{3 \chi_{0}}{\tilde{G}_{1}(\vec{k}, \omega)+\frac{4}{5} \xi^{2} \tilde{G}_{2}^{-1}(\vec{k}, \omega)}\left\{\frac{L(\xi)}{\xi}+\tilde{G}_{2}^{-1}(\vec{k}, \omega)\left[\frac{3}{\xi} L(\xi)-1\right]\right\}
$$

where we have introduced the normalized propagators $\tilde{G}_{1,2}=G_{1,2}(\vec{k}, \omega) / 2 \phi(\omega)$. The dependence of Eqs. (44)-(45) on the dimensionless parameter $\xi$ implies that the response of the system is in general a nonlinear function of the magnitude of the static component $E^{(0)}$. This dependence also influences the characteristic relaxation time as known from experiments 34 .

Our formalism reduces to well known phenomenological relations used to describe dielectric relaxation in diverse materials including solids, glass-forming liquids and polymer melts $\stackrel{14}{ }$, when the appropriate limiting cases are considered.

In particular, Eqs. (44) and (45) can be reduced to the Debye and Cole-Cole ${ }^{21}$ dielectric response functions if the infinite hierachy of dynamic equations for the moments of the distribution, starting with Eqs. (29) and (30), is truncated under the assumption that the order parameter tensor, $S_{i j}$, remains very close to its equilibrium value, i.e. $S_{i j}^{(0)}=S_{i j}^{\text {eq }}\left(\vec{E}^{(0)}\right)$, and $S_{i j}^{(1)}=0$. If in addition we neglect the diffusion of polarization by assuming $\varphi(t)=0$, for all $t$, then Eqs. (44) and (45), reduce to the simpler form

$$
\chi_{\perp, \|}(\omega)=\chi_{\perp, \|}^{o} \frac{2 \phi(\omega)}{i \omega+2 \phi(\omega)}
$$

where the amplitudes of the parallel and perpendicular components, $\chi_{\|}^{o}$ and $\chi_{\perp}^{o}, \operatorname{read}^{29}$

$$
\chi_{\|}^{o}=3 \chi_{0} \frac{L(\xi)}{\xi}, \text { and } \chi_{\perp}^{o}=\frac{3}{2} \chi_{0}\left(1-\frac{L(\xi)}{\xi}\right) .
$$

When the response of the system can be considered instantaneous, we have $\phi(t)=D \delta(t)$, i.e. $\phi(\omega)=D$, and therefore Eq. (46) takes the form of two independent Debye expressions

$$
\chi_{\perp, \|}(\omega)=\chi_{\perp, \|}^{o} \frac{1}{1+i \omega \tau_{0}},
$$

where the relaxation time, $\tau_{0}$, is related with the rotational diffusion coefficient through $\tau_{0}=1 / 2 D$. On the other hand, for the power law memory kernel given by Eq. (15), we have $\phi(\omega)=D^{\alpha}(i \omega)^{1-\alpha}$, and Eq. (46) reduces to two 
expressions of the Cole-Cole type

$$
\chi_{\perp, \|}(\omega)=\chi_{\perp, \|}^{o} \frac{1}{1+\left(i \omega \tau_{c c}\right)^{\alpha}},
$$

where the relaxation time appearing in this expression, $\tau_{c c}$, is given by $\tau_{c c}=1 / 2^{1 / \alpha} D$.

In the following subsections we will analyze in more detail the dependence on the frequency, the normalized dipole electrostatic energy, the rotational diffusion and the diffusion of polarization of the real and imaginary parts of the complex susceptibility, $\chi_{\perp, \|}^{\prime}=\operatorname{Re}\left\{\chi_{\perp, \|}\right\}$ and $\chi_{\perp, \|}^{\prime \prime}=-\operatorname{Im}\left\{\chi_{\perp, \|}\right\}$.

\section{A. The case of Markovian dynamics}

For simplicity, in the following the analysis will be carried out in different successive reduced levels of description in order to better quantify and elucidate the effects due to couplings, diffusion of polarization and the non-Markovian character of the system. With this aim we have subdivided this section into subsections.

\section{Effects due to the coupling with higher order moments}

We will consider first the effects of the coupling of the average polarization with the moments of second and third order on the dielectric susceptibility. From Eqs. (35) and (36), and (44)-(47), it follows that coupling effects are manifested by the terms proportional to $\tilde{G}_{2}^{-1}(\vec{k}, \omega)$, which is the propagator associated with the time and space evolution of $S_{i j}$. Ignoring for convenience the effects associated to diffusion of polarization by making $\varphi(\omega)=0$, and the non-Markovian effects by assuming an instantaneous rotational diffusion kernel, i.e. $\phi(\omega)=D$, the normalized complex susceptibilities perpendicular and parallel to $\vec{E}^{(0)}$, Eqs. (44) and (45), are given by

$$
\begin{aligned}
& \tilde{G}_{1}(\vec{k}, \tilde{\omega})=1+i \tilde{\omega}, \\
& \tilde{G}_{2}(\vec{k}, \tilde{\omega})=3+i \tilde{\omega},
\end{aligned}
$$



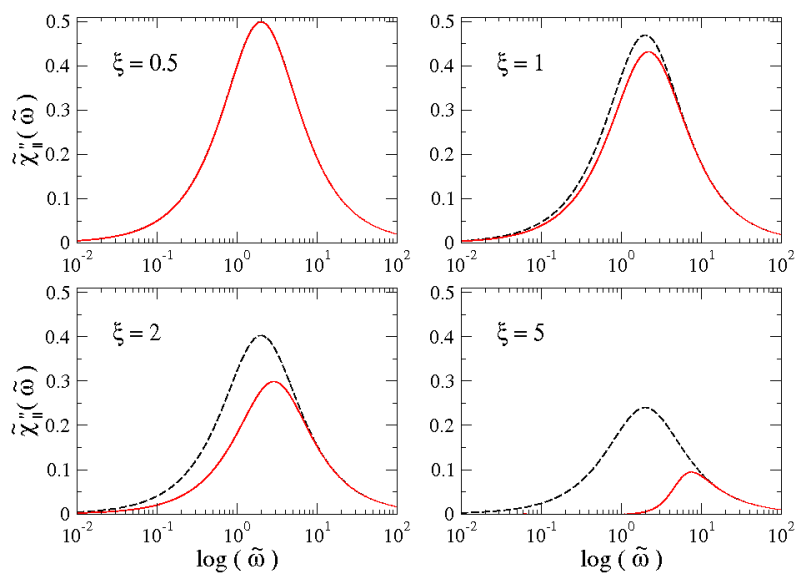

FIG. 1: Imaginary part of the parallel component of the dielectric susceptibility for diverse values of the external field normalized with respect to the average thermal energy, $\xi$. All curves correspond to Markovian processes in the homogeneous approximation. Solid lines contain the effect of the coupling of high order moments of the distribution function as obtained from Eq. 45) with $\tilde{G}_{1}$ and $\tilde{G}_{2}$ given by Eqs. (50) and (51). Dashed lines are obtained from the imaginary part of Eqs. (46) and (47), in which the coupling with high order moments is not taken into account (Debye case).

where the normalized frequency, $\tilde{\omega}$, has been defined by

$$
\tilde{\omega}=\omega / 2 D
$$

When these expressions for $\tilde{G}_{1}$ and $\tilde{G}_{2}$ are used in Eqs. (44) and (45), the result will contain exclusively the effects of the dynamics of the second and third order moments on $\chi_{\perp}$ and $\chi_{\|}$. This result has to be compared with Eq. (48), in which these effects have been neglected. In Figs. 1 1 and 2 we present this comparison for the real and imaginary parts of the normalized susceptibility $\tilde{\chi}_{\|}(\vec{k}, \omega)=\chi_{\|}(\vec{k}, \omega) / \chi_{0}$, and four values of the normalized electrostatic energy, $\xi$, ranging from small, $\xi=0.5$, to large values, $\xi=5$, as compared with the average thermal energy per molecule. It can be observed that when the external static field is small, the coupling with high order moments produce no significant effect on the the complex susceptibility. However, for moderate, $\xi=1.0$, and large external static fields, $\xi=5$, the polar moments of the distribution increase and the changes induced by the coupling between these moments in both $\chi^{\prime}(\omega)$ and $\chi^{\prime \prime}(\omega)$, are appreciable.

Figs. 1 and 2 show that the coupling of higher order moments reduces the susceptibility of the system with respect to the Debye-like case, in which this coupling is neglected. It can be also noticed that the frequency at which $\chi_{\|}^{\prime \prime}$ takes its maximum value is shifted towards the region of large frequencies. However, the frequency dependence of $\chi_{\|}^{\prime \prime}(\omega)$ is not modified in this limit since this quantity still decreases as $\omega^{-1}$. 

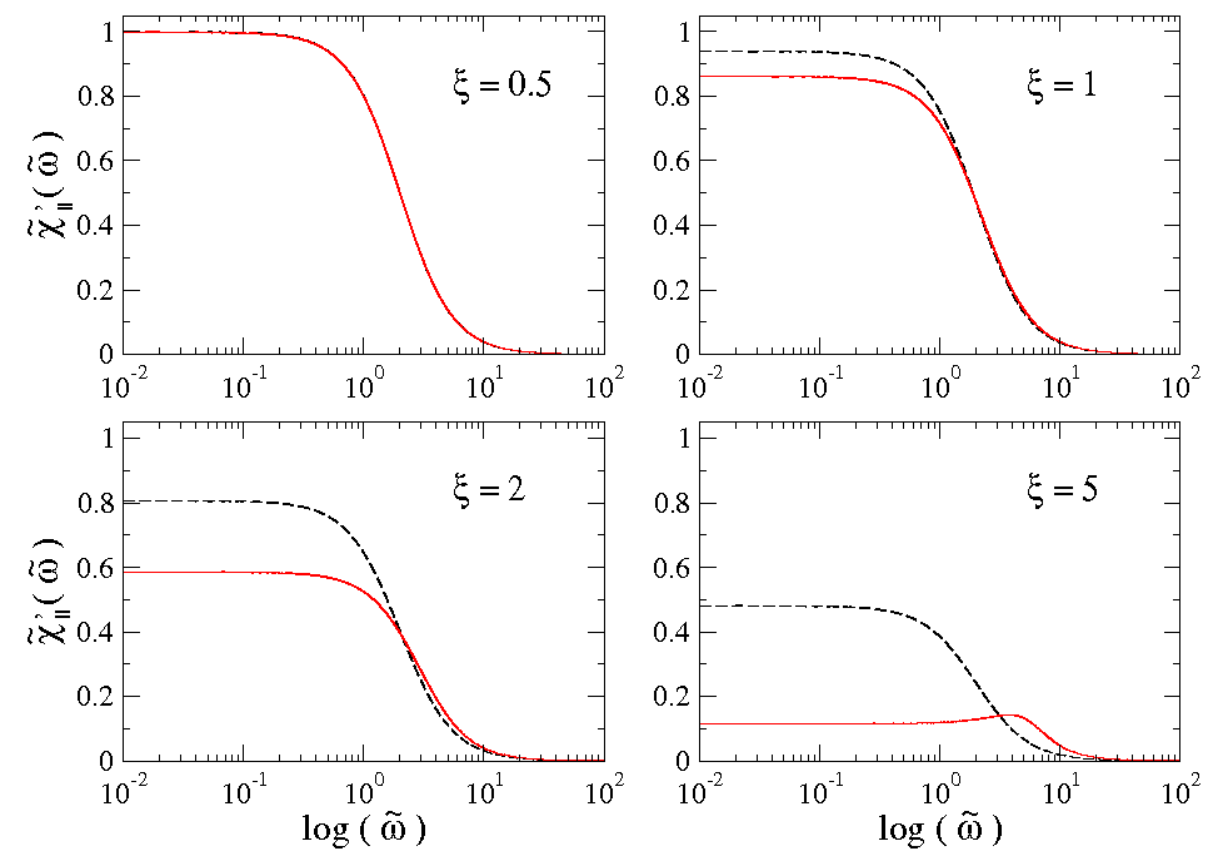

FIG. 2: The same as in Fig. 1 for the real part of the parallel component of the dielectric susceptibility. Solid lines represent the effect of the coupling of high order moments whereas the dashed lines correspond to the Debye case.

\section{Effects due to diffusion of polarization}

The diffusion of polarization may also influence dependence on the wave vector and frequency of the dielectric susceptibility as we will show in this section. In order to focus on these effects, in this case we will consider the Markovian dynamics with memory functions $\phi(\omega)=D$, for the rotational diffusion, and $\varphi(\omega)=\mathcal{D}$, for the diffusion of polarization, that has associated the diffusion coefficient $\mathcal{D}$. We will also ignore the effects due to the coupling of the average dipole moment with moments of second and third order by making $\tilde{G}_{2}^{-1}=0$ in Eqs. (44) and (45).

Within these approximations, only the normalized propagator $\tilde{G}_{1}$ is required for describing the behavior of $\chi_{\perp, \|}$, and it can be written in the form

$$
\tilde{G}_{1}(\vec{k}, \tilde{\omega})=1+\gamma+i \tilde{\omega}
$$

where the parameter $\gamma=k^{2} \mathcal{D} / 2 D$, has been introduced to quantify the relative contribution of the diffusion of polarization with respect to rotational diffusion of the molecules. The normalized frequency $\tilde{\omega}$ is given as before by Eq. (52).

When Eq. (53) together with $\tilde{G}_{2}^{-1}=0$, are used in Eqs. (44) and (45), we obtain expressions which exclusively quantify the effects of the diffusion of polarization on the components of the dielectric susceptibility. Since in this 
approximation the diffusion of polarization only modifies the real part of $\tilde{G}_{1}$, it is clear that its general effect on the imaginary part of $\chi_{\perp}$ and $\chi_{\|}$will consist in decreasing the value and shifting the location of the maxima of these functions in an amount proportional to the parameter $\gamma$. However, it can be noticed that the general dependence of the dielectric susceptibility with respect to the frequency is not modified by a memory kernel of the form $\varphi=\mathcal{D}$, because both $\chi_{\perp}^{\prime \prime}$ and $\chi_{\|}^{\prime \prime}$ increase as $\omega$, for $\tilde{\omega} \ll 1+\gamma$, and decrease as $\omega^{-1}$, for $\tilde{\omega} \gg 1+\gamma$. Similar conclusions are valid for the real part of $\chi_{\perp}$ and $\chi_{\|}$.

\section{B. The case of non-Markovian dynamics}

In this section, we will first consider the effects of the non-instantaneous response of the system to the imposed fields and afterwards both, the effects coming from coupling with higher order moments and those due to the diffusion of polarization. Consequently, we set $\varphi(\omega)=0$, and $G_{2}^{-1}=0$, in Eqs. (44) and (45). This yields Eqs. (46) and (47) for the dielectric susceptibility components, in which the memory kernel, $\phi(\omega)$, has to be given in order to specify the behavior of the dielectric relaxation.

For the sake of clarity, in the present paper, we will only analyze the effects of two types of memory kernels, namely, the power law memory function given by Eq. (15), and a modified kernel that combines both exponential and power law decay. We will write the latter in the form

$$
\phi(t)=\left\{\begin{array}{cc}
\frac{2 D^{2}}{\Gamma(\alpha-1)} e^{-2 D t}(2 D t)^{\alpha-2} & \text { for } t>0 \\
0 & \text { for } t<0
\end{array},\right.
$$

with $0<\alpha<1$.

Let us discuss first the effects of the memory function given by Eq. (15). Previously, we have shown that this function yields the FFPE, Eq. (17), and the Cole-Cole law for the dielectric susceptibility, Eq. (49). Accordingly, the effects of this kind of non-Markovian dynamics can be analyzed by simply comparing our previous results, namely, Eq. (49) with respect to the Debye expression, Eq. (47), in which such effects do not appear.

In Fig. 3 we perform this comparison for the real and imaginary parts of $\chi_{\|}(\omega)$. For convenience, we have illustrated our results in terms of the normalized susceptibility $\tilde{\chi}_{\|}=\chi_{\|} / \chi_{0}$, and the normalized frequency $\tilde{\omega}$, Eq. (52).

Curves in Fig. 3 exhibit the well known features of the Cole-Cole susceptibility. The dielectric peak in the ColeCole case is symmetric and behaves in the form $\chi^{\prime \prime} \sim \omega^{\alpha}, \chi^{\prime \prime} \sim \omega^{-\alpha}$, in the limits of small and large frequencies, respectively. For $0<\alpha<1$, the width of the dielectric loss increases the smaller $\alpha$ is, while the departure with respect 

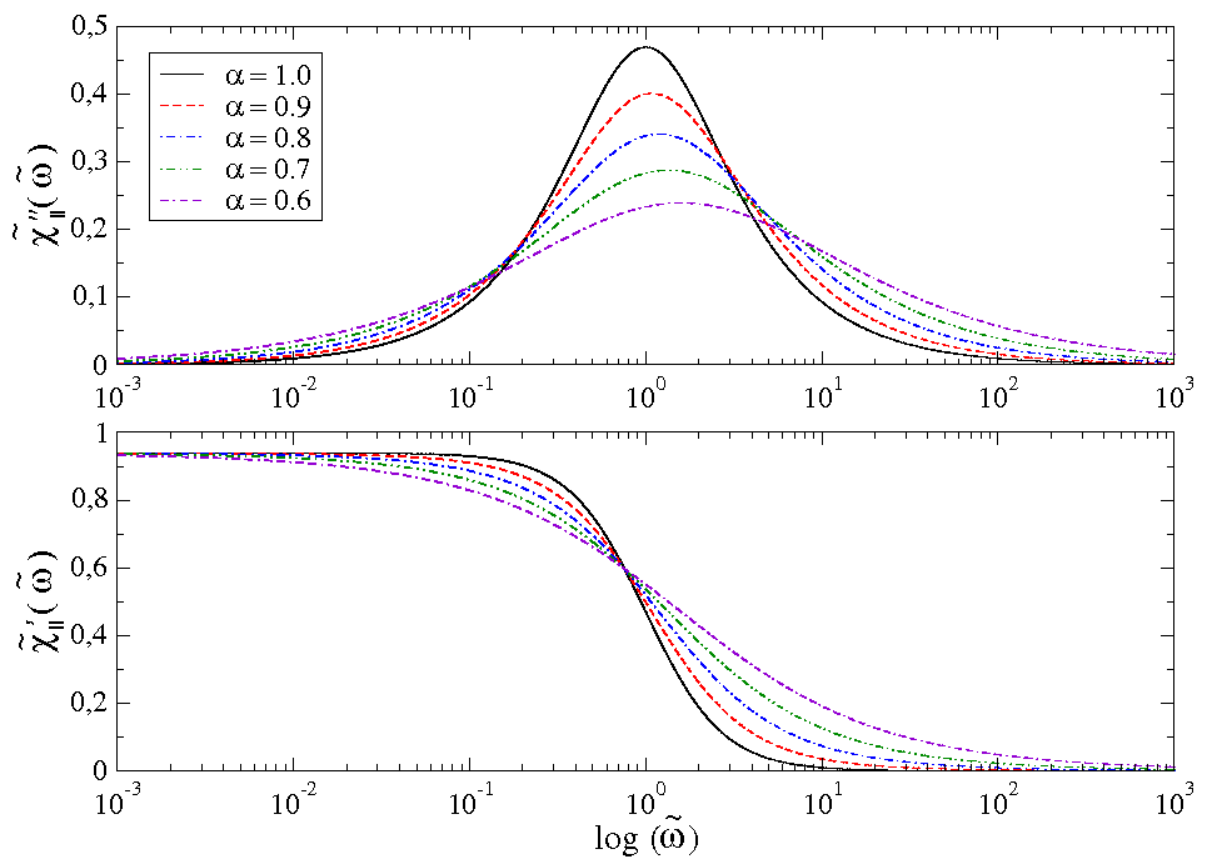

FIG. 3: Normalized parallel component of the dielectric susceptibility in the non-Markovian homogeneous approximation. The memory kernel describing the rotational diffusive processes is considered to decay according to the power law Eq. (15). The dimensionless exponent $\alpha$, takes diverse values including $\alpha=1$, which corresponds to normal diffusion, and $\alpha=0.9,0.8,0.7,0.6$, which describe rotational subdiffusive processes. $\tilde{\chi}_{\|}^{\prime \prime}$ and $\tilde{\chi}_{\|}^{\prime}$ are calculated from Eqs. (49) and (48), with $\tilde{\chi}_{\|}=\chi_{\|} / \chi_{0}$. The value of the normalized field is taken as $\xi=1$.

to the Debye case is eliminated for $\alpha=1$. The frequency dependence of the real part of the susceptibility is also modified by the power law memory function, Eq. (15), and instead of the characteristic Debye's decay, $\chi^{\prime} \sim \omega^{-2}$, a decay of the form $\chi^{\prime} \sim \omega^{-\alpha}$, is observed.

The modified memory function given by Eq. (54) yields an asymmetric shape for the dielectric peak. This result follows from the Fourier transform of Eq. (54): $\phi(\omega)=D(1+i \omega / 2 D)^{1-\alpha}$, and after replacing this expression into Eq. (46). The resulting formula for the normal and parallel components of the susceptibility can be written in the form

$$
\chi_{\perp, \|}(\omega)=\frac{\chi_{\perp, \|}^{o}}{1+i \omega \tau_{0}\left(1+i \omega \tau_{0}\right)^{\alpha-1}}
$$

where we used $\tau_{0}=1 / 2 D$ and the amplitudes $\chi_{\perp, \|}^{o}$ are given by Eq. (47).

In order to analyze the low and high frequency behavior of Eq. (55), we will use the formula

$$
\left(1+i \omega \tau_{0}\right)^{\alpha-1}=\left(1+\omega^{2} \tau_{0}^{2}\right)^{\frac{\alpha-1}{2}} e^{i(\alpha-1) \tan ^{-1}\left(\omega \tau_{0}\right)} .
$$



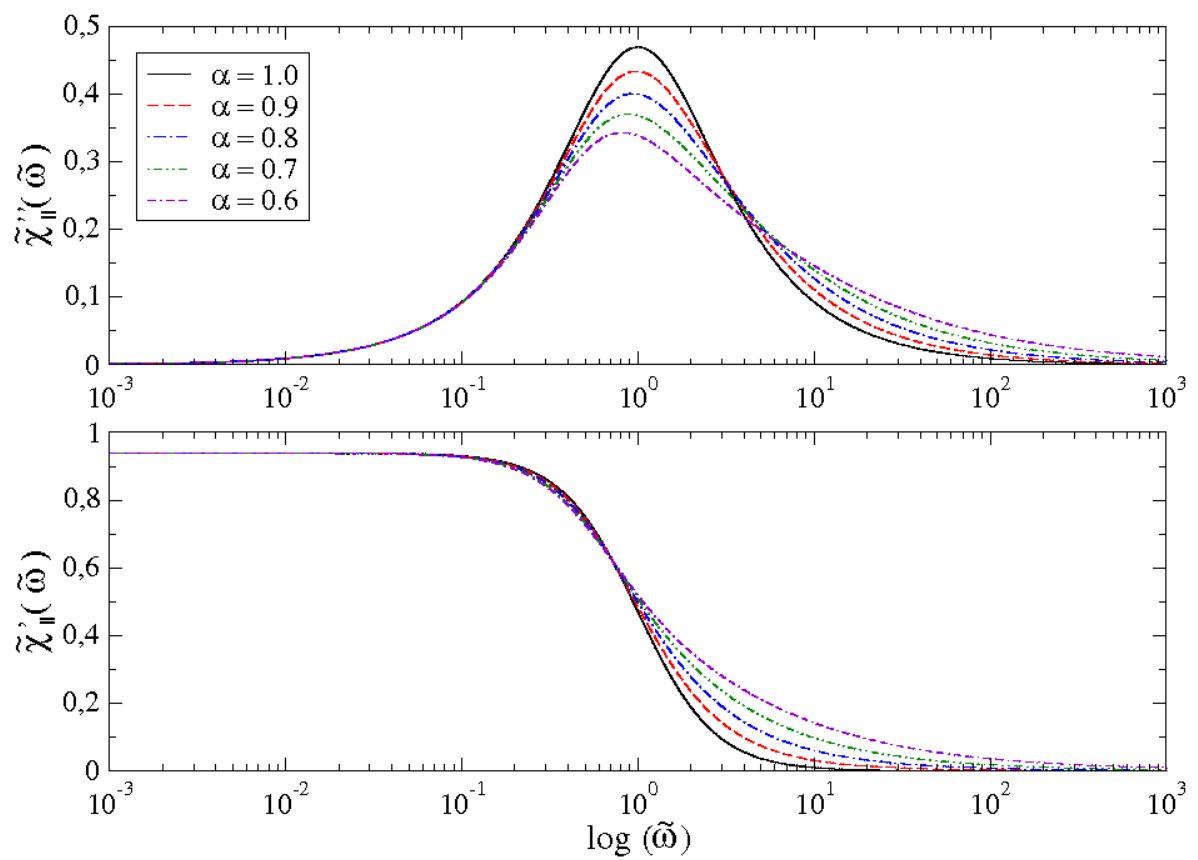

FIG. 4: Normalized parallel component of the dielectric susceptibility in the non-Markovian homogeneous approximation. The memory kernel describing the rotational diffusive processes is considered to decay according to the modified power law Eq. (54). For $\alpha=1$, the usual decay in the dielectric loss $\tilde{\chi}_{\|}^{\prime \prime} \sim \tilde{\omega}^{ \pm 1}$, is observed. For $\alpha<1, \tilde{\chi}^{\prime \prime}$ becomes asymmetric, increasing as $\tilde{\omega}$ and decreasing as $\tilde{\omega}^{-\alpha}$. $\tilde{\chi}_{\|}^{\prime \prime}$ and $\tilde{\chi}_{\|}^{\prime}$ are calculated from Eqs. (55) and (48), with $\tilde{\chi}_{\|}=\chi_{\|} / \chi_{0}$. The value of the normalized field is fixed at $\xi=1$.

In the limit $\omega \tau_{0} \ll 1$, the imaginary part of $\chi_{\perp, \|}$ increases linearly with $\omega$, i.e. $\chi_{\perp, \|} \sim \omega$; while for $\omega \tau_{0} \gg 1$, it decreases as $\chi_{\perp, \|} \sim \omega^{-\alpha}$. This asymmetry in the dielectric loss is not exhibited by the Debye nor by the Cole-Cole functions, but resembles the dependence that is observed in systems described by the phenomenological Cole-Davidson expression $\underline{\underline{14}}$. In a similar way, it can be shown that the real part of $\chi_{\perp, \|}$ decreases as $\chi_{\perp, \|} \sim \omega^{-\alpha}$ for $\omega \tau_{0} \gg 1$, in contrast with the usual Debye dependence. Fig. 4 exhibits these effects on the real and imaginary parts of $\chi_{\|}(\omega)$, in terms of the normalized susceptibility $\tilde{\chi}_{\|}=\chi_{\|} / \chi_{0}$, and the normalized frequency $\tilde{\omega}$, Eq. (52).

The general case in which both, rotational diffusion and the diffusion of polarization processes are present and have a non-Markovian character can be analyzed as a function of the parameter $\xi$. In general terms, the qualitative correction arising from the coupling with higher orders moments only modulates the amplitudes of the obtained functions and their characteristic relaxation times (their location along the $\omega$-axis), as shown in Figs. 1 and 2, Thus, if for simplicity we consider small values of $\xi$, such that the coupling of high order moments is neglected, then the expression for the components of $\chi(\vec{k}, \omega)$ takes the form

$$
\chi_{\perp, \|}(\omega)=\chi_{\perp, \|}^{o} \frac{2 \phi(\omega)}{i \omega+2 \phi(\omega)+k^{2} \varphi(\omega)},
$$


with $\chi_{\perp, \|}^{o}$ given by Eq. (47). When the memory kernels $\phi(t)$ and $\varphi(t)$ are assumed to decay as power laws in time, Eqs. (15) and (18), then Eq. (56) reduces to

$$
\chi_{\perp, \|}(\omega)=\frac{\chi_{\perp, \|}^{o}}{1+\frac{1}{2}\left(\frac{i \omega}{D}\right)^{\alpha}+\frac{k^{2} \mathcal{D}_{\beta} D^{-\beta}}{2}\left(\frac{i \omega}{D}\right)^{\alpha-\beta}},
$$

which, as Eq. (55), is also asymmetric. Unlike in the previous case, now the low and high frequency behaviors of the real and imaginary parts of the susceptibilities will be determined by the exponents $\alpha$ and $\beta$. This result implies that the diffusion of polarization introduces asymmetries in the response functions of the system that depend on the viscoelastic properties of the medium. The representation of Eq. (57) contains a combination of the effects shown in Figs. 3 and 4 ,

\section{Cooperative effects}

A simple and effective generalization of the present model can be formulated along the following lines. Consider the case of a system in which different kinds of polar elements, characterized by their dipolar moments, $p^{(q)}$, are present. In this case, the probability distribution function will depend on several orientation vectors, $\vec{n}^{(q)}$, associated to each type of polar element. For example, in the case $q=2$, the distribution function $f\left(\vec{r}^{(1)}, \vec{r}^{(2)}, \vec{n}^{(1)}, \vec{n}^{(2)}, t\right)$, will satisfy a bivariate conservation equation entirely similar to Eq. (2), but having the corresponding divergence term in the $\left(\vec{r}^{(1)}, \vec{r}^{(2)}, \vec{n}^{(1)}, \vec{n}^{(2)}\right)$-space.

As a consequence, a bivariate Fokker-Planck equation similar to Eq. (13) can be obtained from the mesoscopic thermodynamics analysis. This equation can in turn be used to formulate the corresponding evolution equations for the moments of the distribution, in which $P_{i}^{(q)}(\vec{r}, t)=\mathcal{N}_{q} \int d \vec{n}^{(1)} d \vec{n}^{(2)} d \vec{r}^{(1)} d \vec{r}^{(2)} p_{i}^{(q)} f \delta\left(\vec{r}-\vec{r}^{(q)}\right), q=1,2$, are the dipole moments, see Eq. (20). Here, $\mathcal{N}_{q}$ is the number of polar elements of class $q$, and the operator $\delta\left(\vec{r}-\vec{r}^{(q)}\right)$, evaluates the value of the field $\vec{P}^{(q)}$ in the continuous position $\vec{r}$, see for example $\frac{35}{3}$. The definitions of the quadrupolar and octupolar moments are therefore given by entirely similar relations as those of Eqs. (21) and (22).

If we assume that these polar elements are independent, a simple and powerful model can be formulated to account for experiments. Under the independence assumption, the evolution equations associated with $\vec{P}^{(1)}(\vec{r}, t)$ and $\vec{P}^{(2)}(\vec{r}, t)$, are also independent and therefore, the total relaxation process results from the sum of both contributions. Taking into account only the time dependent field, the relation between the polarization and the applied electric field can be 
written as

$$
P_{i}=\sum_{m=1}^{q} P_{i}^{(m)}(\vec{k}, \omega)=\chi_{i j}(\vec{k}, \omega) E_{j}(\vec{k}, \omega)
$$

where $\chi_{i j}(\vec{k}, \omega)=\sum_{m=1}^{q} \chi_{i j}^{(m)}(\vec{k}, \omega)$, since in the present approximation the response function is independent of the applied field.

As an example, consider that $q=2$ and that the diffusion of polarization effect is subdiffusive anomalous. Thus, by applying a similar procedure to the one yielding Eq. (57), we obtain

$$
\chi(\omega)=\frac{\chi_{1}^{o}}{1+\frac{1}{2}\left(\frac{i \omega}{D_{1}}\right)^{\alpha_{1}}+\frac{k^{2} \mathcal{D}_{\beta_{1}} D_{1}^{-\beta_{1}}}{2}\left(\frac{i \omega}{D_{1}}\right)^{\alpha_{1}-\beta_{1}}}+\frac{\chi_{2}^{o}}{1+\frac{1}{2}\left(\frac{i \omega}{D_{2}}\right)^{\alpha_{2}}+\frac{k^{2} \mathcal{D}_{\beta_{2}} D_{2}^{-\beta_{2}}}{2}\left(\frac{i \omega}{D_{2}}\right)^{\alpha_{2}-\beta_{2}}},
$$

where the subscripts for the parallel and perpendicular components have been suppressed in order to simplify the notation. In this case, the susceptibility is also asymmetric but it may present two broad peaks when the characteristic relaxation times associated to $\vec{P}^{(1)}$ and $\vec{P}^{(2)}$ are sufficiently separated.

The imaginary part of Eq. (59) can be used to account for the dielectric relaxation spectra of glassy materials. This is shown in Fig. 5, where a comparison between experiments (symbols) and theory (lines) is presented in a normalized representation of the dielectric loss, $\bar{\chi}^{\prime \prime}$, see Ref. $\stackrel{36}{.}$. The experiments were performed with $50 / 50$ (wt/wt) glycerol/water samples at $160 \mathrm{~K}$ (solid), in the absence and the presence of myoglobin at different levels of hydration. The symbol $h$ stands for the level of hydration, where $h=\infty$ corresponds to the case when no myoglobin was added. The dielectric relaxation spectroscopy experiments were performed by introducing the samples in a capacitor and applying an oscillatory electric field ${ }^{36}$. The presence of the hydrated myoglobin broadens the spectrum and shifts the maximum towards low frequencies. In Fig. 5 we have included two cases: (a) Without considering diffusion of polarization, i.e. $\mathcal{D}_{\beta_{1}}=\mathcal{D}_{\beta_{2}}=0$, in Eq. (59). (b) Considering the diffusion of polarization, i.e. $\mathcal{D}_{\beta_{1}} \neq \mathcal{D}_{\beta_{2}} \neq 0$. It can be observed that the diffusion of polarization improves the agreement between theoretical and experimental results.

It is interesting to notice that the diffusion of polarization appears to have most dramatic effects in the absence of myoglobin, while its effects are small in the presence of myoglobin. These effects can be understood by considering that the reduction of the amplitude of the dielectric loss is due to the presence of both the myoglobin and the hydration shell, whereas the addition of new modes at low and high frequencies can be attributed to the presence of the hydration shell ${ }^{36}$. Then, it is plausible to assume that the hydration shell induces long-ranged orientational correlations between solvent particles, which reduce the relative importance of the diffusion of polarization term with respect to the non- 

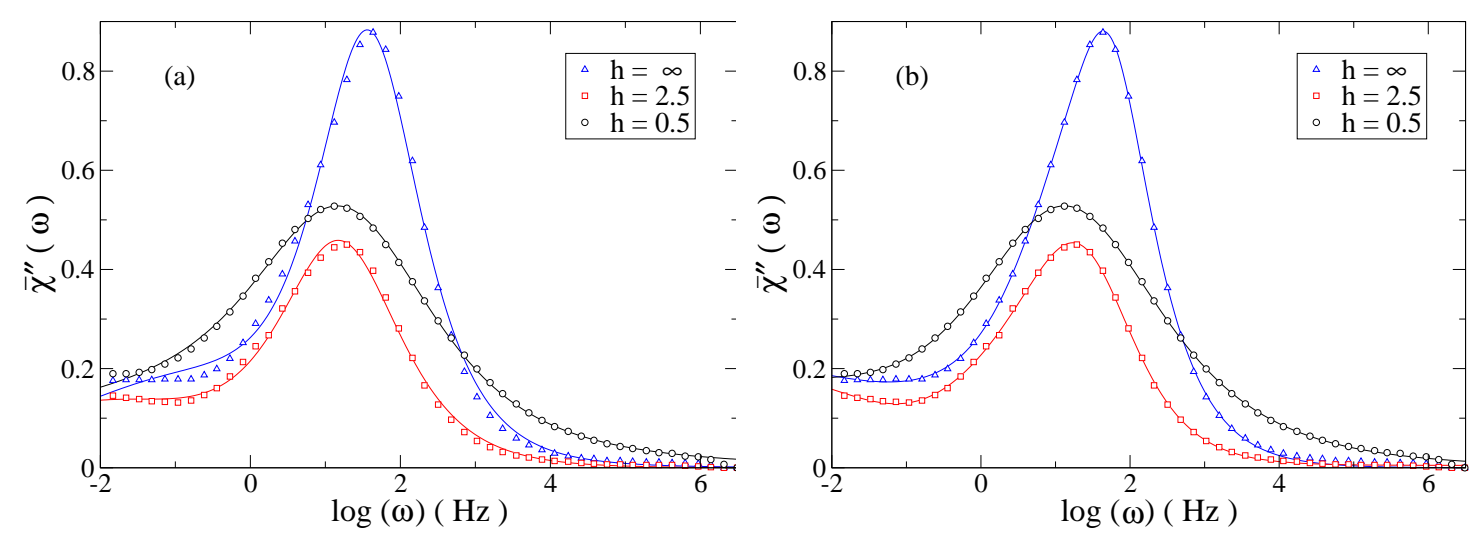

FIG. 5: Normalized dielectric loss of glycerol/water samples at $160 \mathrm{~K}$ in presence of myoglobin with different levels of hydration. The symbols represent experimental results taken from Ref ${ }^{36}$ whereas the lines are the fits from Eq. (59). Our results suggest that the structural $\alpha$-relaxation process affects the observed $\beta$-relaxation spectrum by introducing an asymmetry in the amplitudes of the spectrum at low frequencies.

diffusive terms in all the evolution equations for the multipolar moments. The orientational correlations explain why in Fig. 5 a the agreement for the larger value of the hydration number $(h=2.5)$ is better at low frequencies than in the case of low hydration numbers $(h=0.5)$. For low $h$, the hydration shell is less important and therefore the amplitude of the dielectric loss increases as well as the importance of the diffusion term in the evolution equations for the multipoles.

In this specific application, the superposition of two independent dielectric responses given by Eq. (59), accounts very well for the complicated shape of the dielectric loss found experimentally. According to our model, this function should consist of a peak centered at small frequencies, associated with the so called $\alpha$-relaxation processes, superposed to a peak located at larger frequencies, which is associated with slower or $\beta$-relaxation processes. The latter peak is the one observed in Figs. 5 (a) and (b). It can be noticed that the diffusion of polarization allow for fitting the experimental data in the range of small frequencies. Notice that, as $h$ increases, the exponent $\beta$ increases as well, implying that $\beta(h)$ and indicating that hydration favors a transition from anomalous to normal diffusion of polarization, see the tables in Appendix B.

\section{DISCUSSION}

A physical insight of the mechanism underlying the power-law behavior of the memory kernel in Eq. (15) can be elucidated by decomposing the corresponding spectrum in terms of elementary Debye processes, that is, by appealing to the theory of distribution of relaxation times 37 . For simplicity, our analysis will be focused on the description leading to Cole-Cole parallel susceptibility (49) with the use of the power law memory function, Eq. (15). 
We will proceed by assuming the existence of a distribution of elementary processes with proper relaxation times and amplitudes, whose superposition generates a wider spectrum such that corresponding to the Cole-Cole function, Eq. (49). In this form, the Cole-Cole function can be recovered from a superposition of elementary Debye processes through the relation 38

$$
\chi_{\|}(\omega)=\int_{-\infty}^{\infty} d(\ln |\tilde{\tau}|) \psi(\ln |\tilde{\tau}|) \frac{1}{1+i \omega \tau}=\int_{-\infty}^{\infty} d y \psi(y) \frac{1}{1+i \omega \tau_{c c} e^{y}}
$$

where for simplicity in notation we have introduced the variable $\tilde{\tau}=\tau / \tau_{c c}$ and used the definition $y=\ln |\tilde{\tau}|$ in the second equality. The function $\psi(y)$ is a continuous distribution of relaxation times having the explicit form

$$
\psi(y)=\frac{\chi_{\|}^{o}}{2 \pi} \frac{\sin (\alpha \pi)}{\cosh (\alpha y)+\cos (\alpha \pi)} .
$$

In fact, the analysis can be simplified by considering a discrete expansion of Eqs. (60) and (61), that is, by approximating the Cole-Cole response law by a superposition of independent Debye-like processes $\chi_{k}(\omega)$, distributed over a discrete space in the form

$$
\chi_{\|}(\omega)=\sum_{k=-\infty}^{\infty} \chi_{k}(\omega)=\sum_{k=-\infty}^{\infty} \frac{A_{k}}{1+i \omega \tau_{k}}
$$

where the amplitudes, $A_{k}$, and relaxation times, $\tau_{k}$, of the $k$-th process are given, respectively, by

$$
A_{k}=\frac{\chi_{\|}^{o}}{2 \pi} \frac{\sin (\alpha \pi)}{\cosh (\alpha k)+\cos (\alpha \pi)}, \text { and } \tau_{k}=\tau_{c c} e^{k}
$$

Notice that the index $k$ in the summation of Eq. (63), quantifies the time-scale separation between the $k$-th elementary process and the process with the relaxation time $\tau_{c c}$, which defines the location of the maximum of the dielectric response function. Those processes labeled by an index $k \gg 1$, evolve toward equilibrium in a characteristic time which is much larger than $\tau_{c c}$, while those with $k \ll-1$, decay much faster than the processes with relaxation time of the order of $\tau_{c c}$.

Fig. [6 illustrates the approximation of both the real and imaginary parts of the normalized Cole-Cole function,

$$
\frac{\chi_{\|}^{\prime}(\omega)}{\chi_{\|}^{o}}=\frac{1+\left(\tau_{c c} \omega\right)^{\alpha} \cos (\alpha \pi / 2)}{\left[1+\left(\tau_{c c} \omega\right)^{\alpha}\right]^{2}+\left(\tau_{c c} \omega\right)^{2 \alpha} \sin ^{2}(\alpha \pi / 2)},
$$



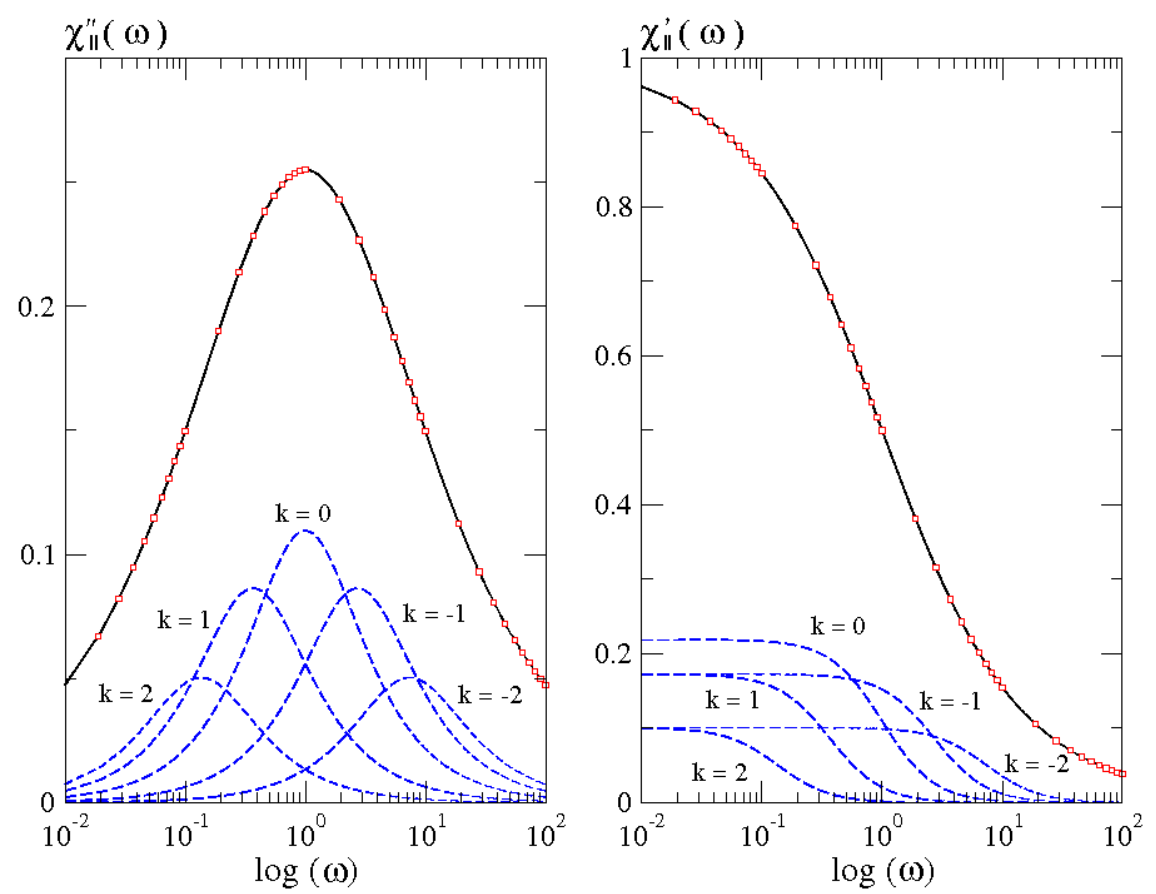

FIG. 6: Approximation of a Cole-Cole function by a superposition of discrete Debye components with amplitudes and relaxation times given by Eq. (63). The real and imaginary part of the Cole-Cole function, Eq. (49), are represented by the continuous curve. They correspond to $\alpha=0.6$. The superposition of Debey components, Eqs. (62) and (63), is represented by the square symbols and it can be observed that it fits the Cole-Cole function over the whole range of frequencies. Some of the Debye components, those with indexes $k=-2,-1,0,1,2$, are explicitly shown.

$$
\frac{\chi_{\|}^{\prime \prime}(\omega)}{\chi_{\|}^{o}}=\frac{\left(\tau_{c c} \omega\right)^{\alpha} \sin (\alpha \pi / 2)}{\left[1+\left(\tau_{c c} \omega\right)^{\alpha}\right]^{2}+\left(\tau_{c c} \omega\right)^{2 \alpha} \sin ^{2}(\alpha \pi / 2)},
$$

by the superposition of Debye components over the discrete distribution of relaxation times given by Eq. (663) with $\alpha=0.6$, and where the infinite sum in Eq. (63) has been approximated by a sum over a finite interval ranging from $k=-100$ to $k=100$. Eq. (63) constitutes a very good approximation to the Cole-Cole function, reproducing very well the behavior of the latter over the whole frequency range considered. It is interesting to notice that a partial superposition of independent Debye contributions in which elementary processes within a given range are suppressed, yields a two step relaxation dynamics manifested through the dielectric loss with well marked peaks as those observed experimentally $\stackrel{39}{\underline{9}}$. This elimination of elementary processes can be represented by non-uniform distributions of characteristic times는.

These results can be used to give an insight of the physical mechanisms underlying the relaxation process if we consider Eqs. (47) and (48), and the definition of the static susceptibility $\chi_{0}=\mathcal{N} p^{2} / 3 k_{B} T$. The amplitude of each elementary Debye contribution in Eq. (62), can be written in terms of an effective numerical density of polar elements 
$\mathcal{N}_{k}$ participating in the process with relaxation time $\tau_{k}$, that is

$$
A_{k}=\frac{\mathcal{N}_{k} p^{2}}{k_{B} T} \frac{L(\xi)}{\xi}
$$

Comparing Eqs. (63) and (66) we obtain

$$
n_{k}=\frac{\xi}{6 \pi L(\xi)} \frac{\sin (\alpha \pi)}{\cosh (\alpha k)+\cos (\alpha \pi)}
$$

where $n_{k}=\mathcal{N}_{k} / \mathcal{N}$, is the effective fraction of polar elements which contribute to process $k$. In the limit of slow and fast processes, i.e. $k \gg 1$ and $k \ll-1$, respectively, and in terms of the relaxation time $\tau_{k}$, Eq. (67), $n_{k}$ can be recast into

$$
n_{k}\left(\tau_{k}\right)=\frac{\xi}{3 \pi L(\xi)} \sin (\alpha \pi)\left\{\begin{array}{l}
\left(\frac{\tau_{k}}{\tau_{c c}}\right)^{\alpha} \text { for } \tau_{k} \ll \tau_{c c} \\
\left(\frac{\tau_{k}}{\tau_{c c}}\right)^{-\alpha} \text { for } \tau_{k} \gg \tau_{c c}
\end{array}\right.
$$

This result implies that the power law memory kernel (15) and consequently the Cole-Cole function (49), can be suitable for describing the dynamics of systems in which a distribution of relaxation times exist, such that the fraction of polar elements of the system participating in the processes with relaxation time $\tau_{k}$, scales as $\tau_{k}^{\alpha}$ in the range of fast processes, while in the range of slow processes it scales as $\tau_{k}^{-\alpha}$. In addition, it should be noticed that the fraction of polar elements participating in a given relaxation process depends on the dimensionless parameter $\xi$ which in turn depends on the static applied field $E^{(0)}$.

\section{CONCLUSIONS}

Starting from purely thermodynamic grounds, we have formulated a very general framework, which is useful for describing non-Debye dielectric relaxation in diverse materials including solids, glass-forming liquids and polymer melts. The framework is based on the derivation of a non-Markovian Fokker-Planck like equation that incorporates memory effects for both rotational diffusion and diffusion of polarization and therefore provides a very wide range of possible behaviors as analyzed through section V. We have shown that fractional Fokker-Planck descriptions can be derived as particular cases of our model when a specific form of the memory kernel is chosen. The formulation of the model on thermodynamic grounds simplifies its generalization to cases in which external temperature or pressure 
gradients, among others, are applied on the system.

The evolution equations for the polarization vector and higher order multipoles can be derived systematically. We analyzed the evolution equations for the first two multipoles by closing the corresponding hierarchy assuming that the higher order multipoles may be considered near to equilibrium. This assumption yields previously known results for the dependence of the susceptibilities and the relaxation times when the system evolves in the presence of a time dependent externally applied electric field consisting of a static and a harmonic dependent component. We analyzed in detail the effect that each mechanism considered has in the behavior of the susceptibilities and shown how empirical formulas can be obtained from the general formalism. In addition, we outlined how the present approach can be generalized to consider the non-Markovian dynamics of several polar elements and the effect of this on the total susceptibility. In this case, our results were used to fit with recent experiments performed with glassy materials damped with myoglobin proteins at different levels of hydration. The agreement between theory and experiment was very good.

The analysis of the susceptibilities by means of the theory of distribution of times lead us to propose a physical interpretation of each relaxation process in terms of the fraction of polar elements that participate in the relaxation process for a given frequency of the external field. We found that this fraction of polar elements, related to a particular characteristic relaxation time, depends on the static externally applied field $E^{(0)}$. This result indicates that the application of a static field may be used to control the fraction of polar elements that participate in a given relaxation process (possibly including multistep relaxation) and thus can be useful in both the analysis and the design of materials with specific absorption windows.

\section{Acknowledgments}

HH acknowledges UNAM-DGAPA for financial support. JGMB thanks CONACYT for financial support and ISH thanks UNAM-DGAPA for partial financial support of Grant No. IN102609.

${ }^{1}$ Y. Yin, S. V. Shiyanovskii, A. B. Golovin, and O. D. Lavrentovich, Phys. Rev. Lett. 95, 087801 (2005).

2 S. Cerveny, R. Bergman, G. A. Schwartz, and P. Jacobsson, Macromolecules 35, 4337 (2002).

3 A. Gadomski, Eur. Phys. J. B 9, 569 (1999).

4 M. H. Vainstein, I. V. L. Costa, R. Morgado, and F. A. Oliveira, Europhys. Lett. 73, 726 (2006). 
${ }^{5}$ R. Hernández, and F. L. Somer, J. Phys. Chem. B, 103, 1064 (1999).

${ }^{6}$ E. Hershkovitz, and R. Hernández, J. Chem. Phys. 122, 014509 (2005).

7 J-L. Dèjardin, Phys. Rev. E 68, 031108 (2003).

8 J-L. Dèjardin, and J. Jadzyn, J. Chem. Phys. 122, 074502 (2005).

${ }^{9}$ K. Funke, S. Brückner, C. Cramer, D. Wilmer, J. Non-Cryst. Solids 921, 307 (2002).

10 S. Kumar, and K. J. Rao, Chemical Physics Letters 387, 91 (2004).

11 W. Kob, J. Phys. Condens. Matter 11, R85 (1999).

12 M. F. Shlesinger, and E. W. Montroll, Proc. Natl. Acad. Sci. USA 81, 1280 (1984).

${ }^{13}$ F. Álvarez, A. Alegría, and J. Colmenero, Phys. Rev. B 44, 7306 (1991).

14 A. K. Jonscher, J. Mat. Sci. 16, 2037 (1981).

15 D. Reguera, J. M. Rubi, and J. M. G. Vilar, J. Phys. Chem. B 109, 21502 (2005).

16 I. Santamaría-Holek, A. Pérez-Madrid, and J. M. Rubi, J. Chem. Phys. 120, 2818 (2004).

17 A. Pérez-Madrid, J. Chem. Phys. 122, 214914 (2005).

18 J. M. Rubi, and A. Pérez-Madrid, Physica A 298, 177 (2001).

19 W. T. Coffey, Y. P. Kalmykov, and J. T. Waldron, The Langevin equation (World Scientific, Singapore, 1996).

${ }^{20}$ P. Talkner, E. Hershkovitz, E. Pollak, and P. Hänggi, Surf. Sci. 437, 198 (1999).

${ }^{21}$ K. S. Cole, and R. H. Cole, J. Chem. Phys. 9, 341 (1941).

22 M. Kröger, and P. Ilg, J. Chem. Phys. 127, 034903 (2007).

23 M. Kröger, and H. S. Sellers, Complex Fluids, Lecture Notes in Physics Vol. 415 (Springer, New York, 1992$)$, pp. 295301.

${ }^{24}$ M. Doi, and S. F. Edwards, The Theory of Polymer Dynamics, (Oxford University, New York, 1998).

${ }^{25}$ R. Kubo, M. Toda, and N. Hashitsume, Statistical Physics II. Nonequilibrium Statistical Mechanics, Second Edition, (Springer, Berlin, 1991).

${ }^{26}$ I. Gyarmati, Non-equilibrium Thermodynamics: Field Theory and Variational Principles, (Springer, Berlin, 1970).

27 R. Zwanzig, Phys. Rev. 124, 983 (1961).

28 I. Santamaría-Holek, J. M. Rubi, and A. Gadomski, J. Phys. Chem. B 111, 2293 (2007).

29 J. G. Méndez-Bermudez, I. Santamaría-Holek, Physica A (2010), doi:10.1016/j.physa.2009.12.058

30 R. Metzler, and J. Klafter, Phys. Rep. 339, 1 (2000).

31 R. Metzler, E. Barkai, and J. Klafter, Phys. Rev. Lett. 82, 3563 (1999).

${ }^{32}$ H. Risken, The Fokker-Planck equation, (Springer, Berlin, 1996).

${ }^{33}$ R. Zwanzig, Nonequilibrium Statistical Mechanics, (University Press, Oxford, 2001).

${ }^{34}$ H. Block, and E. F. Hayes, Trans. Faraday Soc. 66, 2512 (1970) .

35 I. Santamaría-Holek, G. Barrios, and J. M. Rubi, Phys. Rev. E 79, 031201 (2009). 
${ }^{36}$ H. Frauenfelder, G. Chen, J. Berendzen, P. W. Fenimore, H. Jansson, B. H. McMahon, I. R. Stroe, J. Swenson, and R. D. Young, Proc. Natl. Acad. Sci. 106, 5129 (2009).

37 R. Balescu, Statistical Dynamics, (Imperial College, Singapore, 1997).

38 A. Bello, E. Laredo, and M. Grimau, Phys. Rev. B 60, 12764 (1999).

39 K. Adachi, and T. Kotaka, Prog. Polym. Sci. 58, 585 (1993).

${ }^{40}$ H. Híjar, J. G. Méndez-Bermúdez, and I. Santamaría-Holek, 2010 (in progress)

\section{Appendix A: Auxiliar formulae}

In section $\mathrm{V}$, we have discussed how the uniaxial symmetry of the problem reduces the matrices $M_{i j}, N_{i j}$ and $\chi_{i j}$ to diagonal forms that contain two contributions, one parallel and one perpendicular to the static applied field. Making use of Eqs. (38), (39) and (41)-(43), it can be shown that the explicit form of the elements $M_{\perp}, M_{\|}, N_{\perp}$ and $N_{\|}$are given by the expressions:

$$
\begin{gathered}
M_{\perp}(\vec{k}, \omega)=G_{1}(\vec{k}, \omega)+\frac{3}{5} \xi^{2} \frac{\phi^{2}(\vec{k}, \omega)}{G_{2}(\vec{k}, \omega)}, \\
M_{\|}(\vec{k}, \omega)=G_{1}(\vec{k}, \omega)+\frac{4}{5} \xi^{2} \frac{\phi^{2}(\vec{k}, \omega)}{G_{2}(\vec{k}, \omega)}, \\
N_{\perp}(\vec{k}, \omega)=6 \chi_{0} \phi(\vec{k}, \omega)\left\{\frac{1}{2}\left(1-\frac{L(\xi)}{\xi}\right)-\frac{\phi(\vec{k}, \omega)}{G_{2}(\vec{k}, \omega)}\left[-1+\left(\frac{\xi}{2}+\frac{3}{\xi}\right) L(\xi)\right]\right\}, \\
N_{\|}(\vec{k}, \omega)=6 \chi_{0} \phi(\vec{k}, \omega)\left\{\frac{L(\xi)}{\xi}+\frac{2 \phi(\vec{k}, \omega)}{G_{2}(\vec{k}, \omega)}\left[-1+\frac{3}{\xi} L(\xi)\right]\right\} .
\end{gathered}
$$

From these equations, the explicit form of the susceptibilities Eqs. (44) and (45) was calculated. 


\section{Appendix B: Fitting parameters}

The parameters used for fitting the experimental data with Eq. (59) and represented in Figs. 55 (a) and (b), are given in the following table.

\begin{tabular}{|c|c|c|c|c|c|c|c|c|c|c|}
\hline Model & $\chi_{1}^{o}$ & $\chi_{2}^{o}$ & $\alpha_{1}$ & $\alpha_{2}$ & $\beta_{1}$ & $\beta_{2}$ & $\tau_{1}$ & $\tau_{2}$ & $\tau_{\beta_{1}}$ & $\tau_{\beta_{2}}$ \\
\hline Fig. [5 (a) & 1.368 & 0.859 & 0.715 & 0.374 & - & - & 0.063 & 94.470 & - & - \\
\hline Fig. 5. (b) & 4.408 & 0.978 & 0.831 & 0.013 & 0.703 & 0.541 & 0.177 & 0.673 & 0.707 & 0.421 \\
\hline
\end{tabular}

We have introduced the notation $\tau_{q}=1 / 2 D_{q}, \tau_{\beta_{q}}=1 / k^{2} \mathcal{D}_{\beta_{q}}$, for $q=1,2$. 